RESEARCH ARTICLE

\title{
Dry-matter Partitioning and Nutrient Uptake of Rice (Oryza sativa L.) under Modified System of Rice Intensification
}

Sandra Maria Saju* and Thavaprakaash N

Department of Agronomy, Tamil Nadu Agricultural University, Coimbatore - 641003.

Received : $30^{\text {th }}$ December, 2019

Revised : $16^{\text {th }}$ January, 2020

Accepted : $12^{\text {th }}$ February, 2020

\begin{abstract}
A field experiment involving eight treatments with different spacing $(25 \mathrm{~cm}$ row spacing with $25 \mathrm{~cm}, 20 \mathrm{~cm}$, and $15 \mathrm{~cm}$ intra-row spacing; and $20 \mathrm{~cm}$ row spacing with $20 \mathrm{~cm}, 15 \mathrm{~cm}$, and $10 \mathrm{~cm}$ intra-row spacing) and fertilizer levels (100\% and 125\% Recommended Dose Fertilizer - RDF) was conducted in Tamil Nadu Agricultural University, Coimbatore, India during late Samba (September-January) season, 2018-19. The trial aimed to study the effect of high-density planting on dry-matter partitioning and nutrient uptake of rice. The experiment was conducted in a randomized block design with three replications. Results showed that higher dry-matter partitioning of leaf, culm, root and panicle were obtained under closer plant spacing of $20 \mathrm{~cm} \times 10 \mathrm{~cm}$ over other treatments while lower dry-matter accumulation was recorded at wider crop geometry of $25 \mathrm{~cm} \times 25 \mathrm{~cm}$. The nutrient uptake recorded at $25 \mathrm{~cm} \times 15 \mathrm{~cm}$ with $125 \%$ RDF was superior over other treatments followed by $20 \mathrm{~cm} \times 10 \mathrm{~cm}$ with $100 \%$ RDF while the SRI method of planting $(25 \mathrm{~cm} \times 25 \mathrm{~cm})$ recorded lower nutrient uptake. The experimental results inferred that nutrient uptake is more similar to dry-matter. The conventional method of cultivation with $20 \mathrm{~cm} \times 10 \mathrm{~cm}$, produced more dry-matter in all plant parts of rice and foraged higher nutrients compared to all other wider spacing treatments with SRI methodology.
\end{abstract}

Keywords: Modified SRI, high-density planting, dry-matter partitioning, nutrient uptake.

\section{INTRODUCTION}

Rice (Oryza sativa L.) is a primary staple food for a large world population and plays a crucial role in the food security of many countries. Asian countries account for more than ninety per cent of rice production and consumption in the world. India has the largest land area (43.5 mha) under rice with a production of $110 \mathrm{mt}$ (NRRI, 2018). At the current rate of population growth, the demand for rice by the Indian population is targeted to be 115 $\mathrm{mt}$ by the year 2025. The expected trends suggest that under the current scenario of diminishing area, increasing cost of cultivation and dwindling natural resources, India has to produce additionaly $1.7 \mathrm{mt}$ of rice every year. Therefore, the increasing demand has to be satisfied by sustaining a steady increase in production by following appropriate agronomic practices like maintaining optimum plant population and nutrition management, which are significant approaches for increasing the productivity of lowland rice.

The biomass accumulation is having significant influence on the grain yield of rice. However, the productivity of rice is not influenced by the total biomass accumulation, but its partitioning to economic parts contributes to real productivity. Plant density is one of the factor that influence the partitioning of assimilates to the grain. Under dense planting, constraints in the availability and utilization of solar radiation and nutrients by the individual plant result in reduced grain yield. Likewise, under sparse planting, there are inadequate tillers, which leaves space and nutrients unutilized, leading to less number of panicles/unit area and reduced yield. Optimum plant population utilizes resources efficiently and leads to improved dry-matter production resulting in a higher yield of rice (Thavaprakaash et al., 2017). Acquaintance with dry-matter production and nutrient uptake during the crop growth period is a vital aspect of mineral nutrition and in improving rice yield.

Planting of single and young (14 days old) seedlings at a wider square geometry $(25 \mathrm{~cm} \times 25$ $\mathrm{cm}$ ) under SRI method is recommended in India. However, if there is a poor seedling establishment, there is a possibility of poor population. Similarly, poor tillering $\left(16 / \mathrm{m}^{2}\right)$ may also influence the yield reduction in rice. Hence, the SRI methodology needs to be modified with high-density planting, 
which may enhance the yield. Appropriate planting density and nutrient management are important for increasing total dry-matter production and its effective partitioning into panicles, thereby increasing yield. Keeping these points in view, the present investigation was carried out.

\section{MATERIAL AND METHODS}

\section{Location and soil}

A field experiment was conducted to study the effect of high-density planting on dry-matter partitioning and nutrient uptake of rice under a modified System of rice intensification. The experiment was done during late Samba (SeptemberJanuary) season, 2018-19 at Tamil Nadu Agricultural University, Coimbatore $\left(11^{\circ} \mathrm{N}\right.$ and $77^{\circ} \mathrm{E}$ at an altitude of $426.7 \mathrm{~m}$ above MSL and receiving average annual rainfall of $731 \mathrm{~mm}$ ) in the Western agro-climatic zone of Tamil Nadu, India. The soil of the experimental field was clay loam in texture with alkaline $\mathrm{pH}(8.2)$, low in available nitrogen (226.8 $\mathrm{kg} \mathrm{ha}^{-1}$ ), medium in available phosphorus (19.25 $\left.\mathrm{kg} \mathrm{ha}^{-1}\right)$ and high in available potassium (571.1 $\left.\mathrm{kg} \mathrm{ha}^{-1}\right)$.

\section{Treatment details}

The experiment was laid out in a Randomized Block Design (RBD) with eight treatments which were replicated thrice. The treatments are, $T_{1}-25 \mathrm{~cm} \mathrm{x}$ $25 \mathrm{~cm}$ with 100\% Recommended Dose of Fertilizers (RDF) (SRI), $\mathrm{T}_{2}-25 \mathrm{~cm} \times 20 \mathrm{~cm}$ with $100 \% \mathrm{RDF}, \mathrm{T}_{3}$ $25 \mathrm{~cm} \times 15 \mathrm{~cm}$ with $100 \%$ RDF, $\mathrm{T}_{4}-25 \mathrm{~cm} \times 15 \mathrm{~cm}$ with $125 \%$ RDF, $T_{5}-20 \mathrm{~cm} \times 20 \mathrm{~cm}$ with $100 \% \mathrm{RDF}$, $\mathrm{T}_{6}-20 \mathrm{~cm} \times 15 \mathrm{~cm}$ with $100 \% \mathrm{RDF}, \mathrm{T}_{7}-20 \mathrm{~cm} \times 15$ $\mathrm{cm}$ with $125 \% \mathrm{RDF}$ and $\mathrm{T}_{8}$ - Conventional cultivation (transplanting 25-30 days old seedlings at $20 \mathrm{~cm} x$ $10 \mathrm{~cm}, 2-3$ seedlings/hill) with 100\% RDF. Medium duration (130-135 days) Rice variety CO 52 was used for this study.

\section{Rice cultivation}

The recommended dose of fertilizer (RDF) for rice crop (150-50-50 kg ha-1) was applied through urea $(46 \% N)$, single superphosphate (SSP) $(16 \%$ $\left.\mathrm{P}_{2} \mathrm{O}_{5}\right)$ and muriate of potash $\left(60 \% \mathrm{~K}_{2} \mathrm{O}\right)$ as per the treatments. The total amount of SSP and 25 per cent of urea and muriate of potash were applied as basal, whereas the remaining dose was topdressed in equal splits at 50,70, and 100 days after transplanting (DAT). All other cultivation practices were followed as per CPG (2012).

\section{Field observation and laboratory analysis}

Five plants were taken from sampling rows for recording the dry-matter production (DMP) at active tillering, panicle initiation, flowering and maturity stages. The plant samples were collected with utmost care not to damage the roots. The samples were cleaned, separated into leaves, culm, roots, and panicle, shade dried, and then oven-dried at $70 \pm 5^{\circ} \mathrm{C}$ until a constant weight is obtained and dry weight of leaves, culm, roots, and panicle were recorded separately in $\mathrm{kg} \mathrm{ha}^{-1}$. Total dry weight was calculated as the sum of dry weights of the plant components.

For plant analysis, the plants selected for DMP were powdered well by Willy mill and analyzed for different nutrients as per the standard procedures. The nutrient uptake by the leaf, culm, root and panicles were calculated separately at active tillering, panicle initiation, flowering and maturity stages and expressed in $\mathrm{kg} \mathrm{ha}^{-1}$ as follows.

\begin{tabular}{|c|c|c|}
\hline \multirow{2}{*}{$\begin{array}{l}\text { Nutrient uptake } \\
\text { by leaf (kg ha) }\end{array}$} & \multirow[t]{2}{*}{$=$} & $\begin{array}{l}\text { Percentage of nutrient } \mathrm{x} \text { weight } \\
\text { of drymatter of leaf }\left(\mathrm{kg} \mathrm{ha}^{-1}\right)\end{array}$ \\
\hline & & 100 \\
\hline \multirow{2}{*}{$\begin{array}{l}\text { Nutrient uptake } \\
\text { by culm (kg ha) }\end{array}$} & \multirow[t]{2}{*}{$=$} & $\begin{array}{l}\text { Percentage of nutrient } \mathrm{x} \text { weight } \\
\text { of drymatter of culm }\left(\mathrm{kg} \mathrm{ha}^{-1}\right)\end{array}$ \\
\hline & & 100 \\
\hline \multirow[t]{2}{*}{$\begin{array}{l}\text { Nutrient uptake } \\
\text { by root (kg ha) }\end{array}$} & \multirow[t]{2}{*}{$=$} & $\begin{array}{l}\text { Percentage of nutrient x weight } \\
\text { of drymatter of root }\left(\mathrm{kg} \mathrm{ha}^{-1}\right)\end{array}$ \\
\hline & & 100 \\
\hline \multirow{2}{*}{\multicolumn{2}{|c|}{$\begin{array}{l}\text { Nutrient uptake by } \\
\text { panicle (kg ha) }\end{array}$}} & $\begin{array}{l}\text { Percentage of nutrient } x \text { weight } \\
\text { of drymatter of panicle }\left(\mathrm{kg} \mathrm{ha}^{-1}\right)\end{array}$ \\
\hline & & 100 \\
\hline
\end{tabular}

The data recorded were analyzed following standard statistical procedure as described by Gomez and Gomez (2010) to draw valid conclusions.

\section{RESULTS AND DISCUSSION}

\section{Dry-matter production and partitioning}

Dry-matter production reveals the efficacy of the crop to utilize the available resources. A steady increase in dry-matter production was noticed at all crop growth stage. Data on dry-matter production (Table 1) shows distinguishable variation due to spacing and fertilizer treatments.

At the active tillering stage, total dry-matter production was significantly higher (5377 $\mathrm{kg} \mathrm{ha}^{-1}$ ) under the crop geometry of $20 \mathrm{~cm} \times 15 \mathrm{~cm}$ fertilized with $100 \%$ RDF $\left(\mathrm{T}_{6}\right)$ over other spacing levels. SRI planting ( $25 \mathrm{~cm} \times 25 \mathrm{~cm}$ with $100 \% \mathrm{RDF})$ registered markedly lower total dry-matter production, which was statistically identical to $25 \mathrm{~cm} \times 20 \mathrm{~cm}$ and conventional method $(20 \mathrm{~cm} \times 10 \mathrm{~cm})$ of planting both provided with $100 \%$ RDF. Leaf, culm, and root DMP was also in a similar pattern (Figure 1a).

At panicle initiation stage, total dry-matter production recorded was significantly higher (6126 $\mathrm{kg} \mathrm{ha}^{-1}$ ) under $20 \mathrm{~cm} \times 10 \mathrm{~cm}\left(\mathrm{~T}_{8}\right)$ and was statistically similar to $20 \mathrm{~cm} \times 15 \mathrm{~cm}$ at 100\% RDF while SRI method of planting $\left(T_{1}\right)$ recorded distinctly lower total dry-matter production than other treatments. Similar results was obtained for leaf and culm dry-matter partitioning at panicle initiation stage also. (Figure 1b). The root biomass of rice at the panicle initiation 
stage was not significantly altered during the study. At flowering stage, same results was obtained. (Figure 1c).
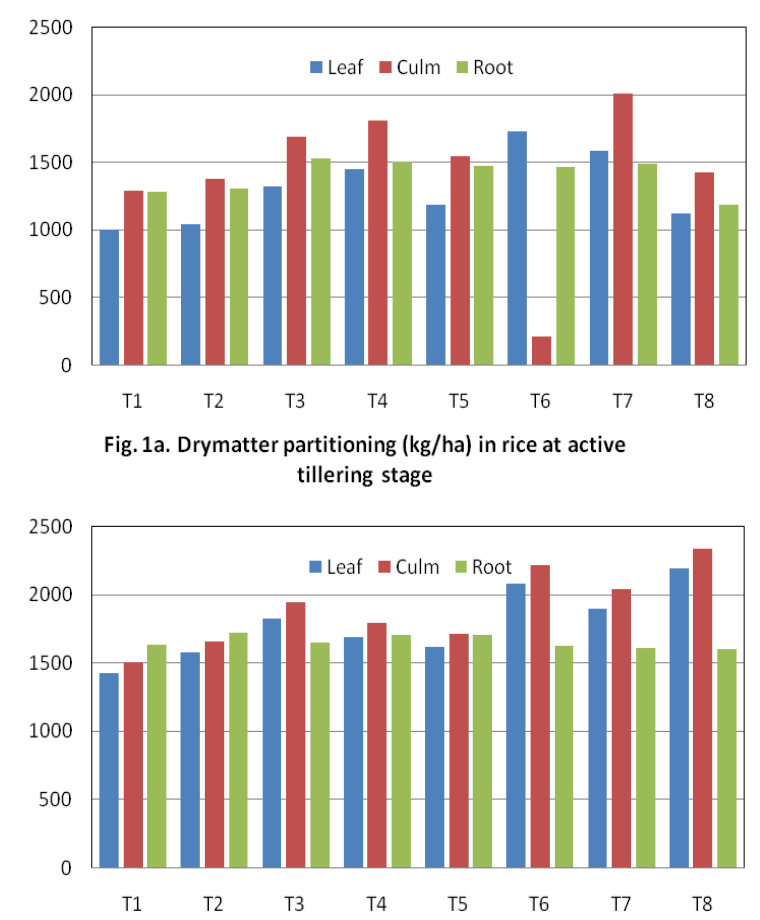

Fig. 1b. Drymatter partitioning $(\mathrm{kg} / \mathrm{ha})$ in rice at panicle initiation stage

At maturity stage, significantly higher total dry-matter production ( $15800 \mathrm{~kg} \mathrm{ha}^{-1}$ ) was obtained under conventional planting system $\left(\mathrm{T}_{8}\right)$ compared to all other treatments except with $20 \mathrm{~cm} \times 15 \mathrm{~cm}$ at $100 \% \mathrm{RDF}, 20 \mathrm{~cm} \times 15 \mathrm{~cm}$ at $125 \% \mathrm{RDF}, 25 \mathrm{~cm}$ $x 15 \mathrm{~cm}$ at $100 \%$ RDF, $20 \mathrm{~cm} \times 20 \mathrm{~cm}$ and $25 \mathrm{~cm} \mathrm{x}$ $15 \mathrm{~cm}$ at $125 \%$ RDF. A significantly lower dry-matter production was obtained under the SRI method of planting $\left(T_{1}\right)$ which was statistically identical to

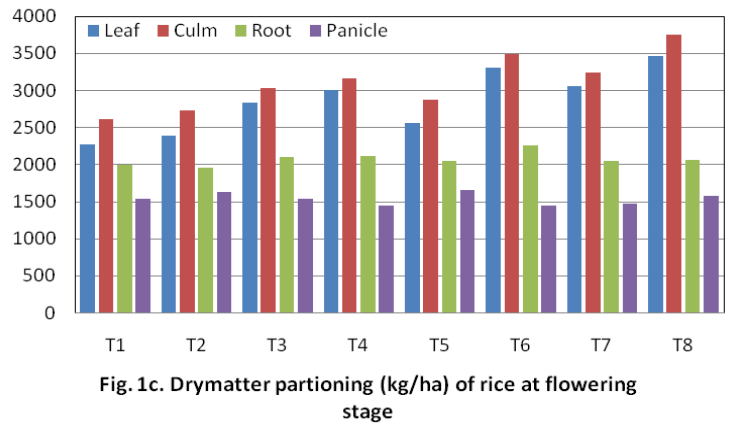

$25 \mathrm{~cm} \times 20 \mathrm{~cm}$. Similar results were noted with leaf and culm dry-matter partitioning (Figure 1d). Panicle dry-matter production was significantly higher (7028 $\mathrm{kg}$ ha) under $20 \mathrm{~cm} \times 20 \mathrm{~cm}\left(\mathrm{~T}_{5}\right)$, which maintained statistical parity with the spacing level of $25 \mathrm{~cm} \times 15$ $\mathrm{cm}$ at both $100 \%$ RDF and $125 \%$ RDF and $25 \mathrm{~cm} \mathrm{x}$ $20 \mathrm{~cm}$ than other treatments. A significantly lower panicle yield was obtained under the conventional system $\left(T_{8}\right)$, which accounted for 37 per cent of total dry-matter production.
Higher dry-matter production at a closer spacing of $20 \mathrm{~cm} \times 10 \mathrm{~cm}$ in most of the growth stages is due to increased plant population, higher nutrient uptake and higher leaf area index over the other spacing levels (Thavaprakaash et al., 2017). Similar results were reported by Najibullah and Chinnusamy (2017). Vigorous growth of individual plant under wider spacing could not compensate the increased number of plants per unit area under closer spacing.

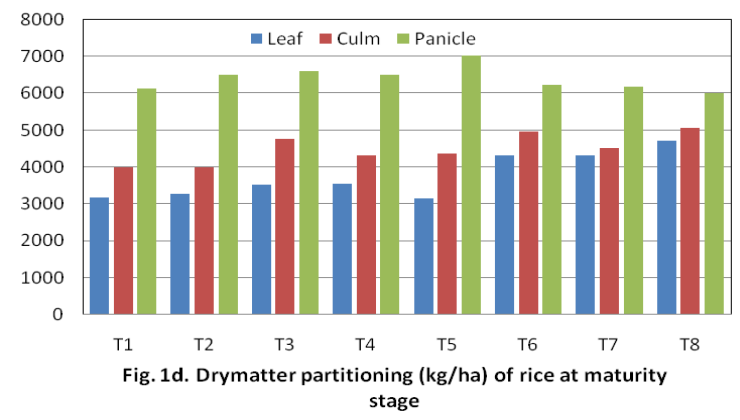

Lower dry-matter production of conventional planting at the active tillering stage can be ascribed to the late transplanting of crop after the advancement of fourth phyllochron. This result is consistent with the earlier findings reported by Aggarwal and Singh (2015).

The dry-matter partitioning recorded was higher for culm and leaf in the earlier growth stages, while reproductive parts contributed to a major proportion of dry-matter production at maturity stage. There is an increased dry-matter accumulation in culm, up to flowering stage and declined thereafter under all the spacing levels. Similar trend was observed in leaf biomass partitioning. The reduction in drymatter of leaf and culm occurred due to larger sink size and effective translocation of photosynthates from rice leaves, sheaths, and culms to grains due to favourable climatic conditions during the grain-filling stage. Dass and Chandra (2013) also reported that leaf dry-matter at maturity stage was comparatively lesser than panicle and culm drymatter accumulation.

Root dry weight also increased up to flowering stage thereafter declined due to degeneration of roots. Thakur et al. (2010) stated that under wider spacing during maturing stage, individual hill produced higher root dry weight, developed more xylem exudates, which transported towards shoot at quicker rates contributing to higher chlorophyll, produced better yield attributes and grain yield for an individual hill.

\section{Nutrient uptake}

Nutrient uptake gradually increased with an increase in crop age (Table 2, 3, and 4). At the active tillering stage, crop geometry of $25 \mathrm{~cm} \times 15 \mathrm{~cm}$ at $125 \% \operatorname{RDF}\left(\mathrm{T}_{4}\right)$ registered comparatively higher total $\mathrm{N}$ 
Table 1. Effect of high-density planting on dry-matter production $\left(\mathrm{kg} \mathrm{ha}^{-1}\right)$ of rice under the modified system of rice intensification

\begin{tabular}{|c|c|c|c|c|c|}
\hline Treatments & $\begin{array}{r}\text { Active tillering } \\
\text { stage }\end{array}$ & $\begin{array}{r}\text { Panicle initiation } \\
\text { stage }\end{array}$ & $\begin{array}{r}\text { Flowering } \\
\text { stage }\end{array}$ & Maturity & stage \\
\hline T1: $25 \mathrm{~cm} \times 25 \mathrm{~cm}+100 \% \mathrm{RDF}$ & 3573 & 4567 & 8414 & & 13329 \\
\hline T2: $25 \mathrm{~cm} \times 20 \mathrm{~cm}+100 \%$ RDF & 3720 & 4945 & 8710 & & 13800 \\
\hline T3: $25 \mathrm{~cm} \times 15 \mathrm{~cm}+100 \%$ RDF & 4531 & 5418 & 9512 & & 14904 \\
\hline T4: $25 \mathrm{~cm} \times 15 \mathrm{~cm}+125 \%$ RDF & 4765 & 5182 & 9735 & & 14376 \\
\hline T5: $20 \mathrm{~cm} \times 20 \mathrm{~cm}+100 \% \mathrm{RDF}$ & 4202 & 5032 & 9146 & & 14551 \\
\hline T6: $20 \mathrm{~cm} \times 15 \mathrm{~cm}+100 \% \mathrm{RDF}$ & 5377 & 5912 & 10488 & & 15475 \\
\hline T7: $20 \mathrm{~cm} \times 15 \mathrm{~cm}+125 \%$ RDF & 5089 & 5536 & 9832 & & 15036 \\
\hline T8: $20 \mathrm{~cm} \times 10 \mathrm{~cm}+100 \%$ RDF & 3736 & 6126 & 10863 & & 15800 \\
\hline SEd & 202 & 250 & 446 & & 668 \\
\hline$C D(P=0.05)$ & 432 & 537 & 957 & & 1431 \\
\hline
\end{tabular}

uptake $\left(82.91 \mathrm{~kg} \mathrm{ha}^{-1}\right)$, total $\mathrm{P}$ uptake $(18.80 \mathrm{~kg}$ $\mathrm{ha}^{-1}$ ) and $\mathrm{K}$ uptake (75.58 $\left.\mathrm{kg} \mathrm{ha}^{-1}\right)$ over all other treatments. Significantly lower total N, P and K uptake was obtained under SRI method of planting $\left(T_{1}\right)$. Nitrogen uptake by leaf was recorded to be distinctly higher (37.66 kg ha-1) under $25 \mathrm{~cm} \mathrm{x}$ $15 \mathrm{~cm}$ plant spacing with $125 \%$ RDF $\left(\mathrm{T}_{4}\right)$ while lower leaf $\mathrm{N}$ uptake was obtained in a wider plant spacing of $25 \mathrm{~cm} \times 25 \mathrm{~cm}$ with $100 \%$ RDF $\left(\mathrm{T}_{1}\right)$ but was statistically equal to $T_{2}(25 \mathrm{~cm} \times 20 \mathrm{~cm}+100 \%$ RDF). Similar results was noticed in the case of rice culm and root. Nitrogen accumulation in roots was lower compared to leaf and culm,phosphorus and potassium uptake by leaf and culm at the active tillering stage were also showed similar trend.

Table 2. Effect of high-density planting on nitrogen uptake $\left(\mathrm{kg} / \mathrm{ha}^{-1}\right)$ of rice under the modified system of rice intensification

\begin{tabular}{|c|c|c|c|c|c|c|c|c|c|c|c|c|c|c|c|c|c|}
\hline \multirow[t]{2}{*}{ Treatments } & \multicolumn{4}{|c|}{ Active tillering stage } & \multicolumn{5}{|c|}{ Panicle initiation stage } & \multicolumn{6}{|c|}{ Flowering stage } & \multicolumn{2}{|c|}{ Maturity stage } \\
\hline & Leaf & Culm & Root & Total & Leaf & Culm & Root & Total & Leaf & Culm & Root & Panicle & Total & Leaf & Culm & Panicle & Total \\
\hline $\mathrm{T}_{1}$ & 21.01 & 20.64 & 6.54 & 48.19 & 24.08 & 24.11 & 8.17 & 56.37 & 30.44 & 33.91 & 9.57 & 20.48 & 94.40 & 16.2 & 20.4 & 83.0 & 119.6 \\
\hline $\mathrm{T}_{2}$ & 22.20 & 24.78 & 7.03 & 54.00 & 28.31 & 28.16 & 8.58 & 65.05 & 35.93 & 37.89 & 9.80 & 22.80 & 106.42 & 16.5 & 20.8 & 91.2 & 128.5 \\
\hline $\mathrm{T}_{3}$ & 31.64 & 30.34 & 8.40 & 70.38 & 34.69 & 33.94 & 9.91 & 78.55 & 42.52 & 42.18 & 10.51 & 21.57 & 116.78 & 17.3 & 23.4 & 92.4 & 133.1 \\
\hline $\mathrm{T}_{4}$ & 37.66 & 36.21 & 9.03 & 82.91 & 37.72 & 37.65 & 11.76 & 87.14 & 51.14 & 47.77 & 13.10 & 22.92 & 134.93 & 18.4 & 23.7 & 97.5 & 139.6 \\
\hline$T_{5}$ & 29.51 & 29.38 & 7.65 & 66.53 & 33.92 & 29.08 & 11.09 & 74.09 & 39.69 & 40.33 & 11.47 & 23.18 & 114.68 & 16.1 & 21.8 & 99.1 & 137.0 \\
\hline$T_{6}$ & 36.25 & 35.02 & 5.85 & 77.12 & 37.35 & 36.58 & 7.29 & 81.22 & 49.56 & 45.27 & 11.73 & 20.26 & 126.81 & 20.7 & 22.8 & 86.9 & 130.4 \\
\hline $\mathrm{T}_{7}$ & 34.93 & 34.17 & 6.11 & 75.22 & 35.03 & 35.65 & 8.03 & 78.71 & 47.70 & 46.07 & 12.33 & 21.97 & 128.08 & 21.7 & 23.5 & 89.8 & 134.9 \\
\hline $\mathrm{T}_{8}$ & 23.99 & 25.69 & 5.82 & 55.50 & 39.26 & 38.51 & 7.51 & 85.28 & 51.29 & 50.59 & 10.32 & 22.20 & 134.40 & 23.6 & 24.8 & 90.3 & 138.7 \\
\hline SEd & 1.40 & 1.34 & 0.30 & 2.90 & 1.60 & 1.60 & 0.40 & 3.50 & 2.10 & 2.00 & 0.50 & 0.95 & 5.50 & 0.9 & 1.0 & 3.9 & 5.6 \\
\hline$C D(P=0.05)$ & 2.90 & 2.90 & 0.60 & 6.40 & 3.30 & 3.40 & 0.80 & 7.30 & 4.50 & 4.40 & 1.07 & 2.03 & 11.90 & 2.0 & 2.2 & 8.4 & 12.1 \\
\hline
\end{tabular}

RDF- Recommended Dose of Fertilizer (150:50:50 N:P:K kg ha-1) NS - Non-significant

\begin{tabular}{|c|c|c|}
\hline T1 & $25 \times 25 \mathrm{~cm}+100 \%$ RDF & T5 \\
\hline T2 & $25 \times 20 \mathrm{~cm}+100 \%$ RDF & T6 \\
\hline T3 & $25 \times 15 \mathrm{~cm}+100 \%$ RDF & $\mathrm{T} 7$ \\
\hline T4 & $25 \times 15 \mathrm{~cm}+125 \% \mathrm{RDF}$ & T8 \\
\hline
\end{tabular}

At panicle initiation stage, significantly higher total $\mathrm{N}$ uptake $\left(87.14 \mathrm{~kg} \mathrm{ha}^{-1}\right)$ was recorded at the spacing level of $25 \mathrm{~cm} \times 15 \mathrm{~cm}$ with $100 \% \operatorname{RDF}\left(\mathrm{T}_{3}\right)$, and SRI method of planting $\left(\mathrm{T}_{1}\right)$ registered a lower $\mathrm{N}$ accumulation than other treatments. Discernibly higher total $\mathrm{P}$ and $\mathrm{K}$ uptake were recorded at the plant spacing of $25 \mathrm{~cm} \times 15 \mathrm{~cm}$ with $125 \%$ RDF $\left(\mathrm{T}_{4}\right)$, and a lower $\mathrm{P}$ and $\mathrm{K}$ uptake were noted in SRI method of planting $\left(T_{1}\right)$. Significantly higher leaf $N$ uptake (39.26 kg ha) was estimated in conventional planting $\left(\mathrm{T}_{8}\right)$. However, a wider spacing level of $25 \mathrm{~cm} \times 25 \mathrm{~cm}$ fed with $100 \% \operatorname{RDF}\left(\mathrm{T}_{1}\right)$ recorded the lowest leaf $\mathrm{N}$ accumulation at the panicle initiation stage. Similar results were replicated in leaf and culm N, P, and $\mathrm{K}$ uptake for different treatments. Comparatively higher $\mathrm{N}$ uptake by root $(11.76 \mathrm{~kg}$
$20 \times 20 \mathrm{~cm}+100 \%$ RDF $20 \times 15 \mathrm{~cm}+100 \% \mathrm{RDF}$ $20 \times 15 \mathrm{~cm}+125 \% \mathrm{RDF}$ $20 \times 10 \mathrm{~cm}+100 \%$ RDF

$\mathrm{ha}^{-1}$ ) at the panicle initiation stage was recorded under plant spacing of $25 \mathrm{~cm} \times 15 \mathrm{~cm}$ at $125 \%$ RDF $\left(T_{4}\right)$ over other treatments. Significantly lower root $\mathrm{N}$ uptake was observed under $20 \mathrm{~cm} \times 15 \mathrm{~cm}$ plant spacing with $100 \% \operatorname{RDF}\left(\mathrm{T}_{6}\right)$. P and $\mathrm{K}$ uptake by roots were also almost in similar trend. At flowering stage, similar nutrient uptake pattern was noted.

Total uptake of nutrients at maturity stage was significantly higher at a crop geometry of $25 \mathrm{~cm} x$ $15 \mathrm{~cm}$ with $125 \% \operatorname{RDF}\left(\mathrm{T}_{4}\right)$, and discernibly lower total uptake was recorded at SRI method of planting $\left(\mathrm{T}_{1}\right)$ for $\mathrm{N}, \mathrm{P}$, and $\mathrm{K}$. At maturity stage, $\mathrm{N}, \mathrm{P}$ and $\mathrm{K}$ accumulation by leaf were found to be higher under conventional planting $\left(\mathrm{T}_{8}\right)$ over other treatments. Planting density of $20 \mathrm{~cm} \times 20 \mathrm{~cm}$ applied with $100 \% \operatorname{RDF}\left(T_{5}\right)$ registered a lower leaf $N$ uptake, and 
it was statistically similar to SRI method $\left(T_{1}\right)$, while $\mathrm{P}$ and $\mathrm{K}$ uptake were recorded to be lesser under wider spacing of $25 \mathrm{~cm} \times 25 \mathrm{~cm}$ with $100 \%$ RDF $\left(\mathrm{T}_{1}\right)$. Higher $\mathrm{N}, \mathrm{P}$, and $\mathrm{K}$ accumulation by culm was recorded at conventional planting $\left(\mathrm{T}_{8}\right)$ and lower at SRI planting method. Significantly higher N uptake by panicle was recorded at a spacing level of $20 \mathrm{~cm}$ x $20 \mathrm{~cm}$ applied with 100\% RDF $\left(\mathrm{T}_{5}\right)$, and lowest panicle $\mathrm{N}$ uptake was obtained in the SRI method $\left(\mathrm{T}_{1}\right)$. Panicle $\mathrm{P}$ and $\mathrm{K}$ uptake were registered to be higher under $25 \mathrm{~cm} \times 15 \mathrm{~cm}$ at $125 \%$ of RDF $\left(T_{4}\right)$ overall treatments except $\mathrm{T}_{5}(20 \mathrm{~cm} \times 20 \mathrm{~cm}$ with 100\% RDF). Significantly lower P uptake by panicle was recorded at the closer spacing level of $20 \mathrm{~cm} \mathrm{x}$ $10 \mathrm{~cm}$ applied at $100 \%$ of RDF $\left(T_{8}\right)$.

Table 3. Effect of high-density planting on phosphorus uptake (kg ha-1) of rice under the modified system of rice intensification

\begin{tabular}{|c|c|c|c|c|c|c|c|c|c|c|c|c|c|c|c|c|c|}
\hline \multirow{2}{*}{ Treatments } & \multicolumn{4}{|c|}{ Active tillering stage } & \multicolumn{4}{|c|}{ Panicle initiation stage } & \multicolumn{5}{|c|}{ Fowering stage } & \multicolumn{4}{|c|}{ Maturity stage } \\
\hline & Leaf & Culm & Root & Total & Leaf & Culm & Root & Total & Leaf & Culm & Root & Panicle & Total & Leaf & Culm & Panicle & Total \\
\hline $\mathrm{T}_{1}$ & 3.60 & 4.64 & 4.60 & 12.85 & 4.82 & 4.82 & 5.48 & 15.12 & 6.25 & 7.33 & 5.78 & 4.33 & 23.69 & 7.17 & 8.32 & 11.68 & 27.16 \\
\hline $\mathrm{T}_{2}$ & 3.80 & 5.16 & 4.82 & 13.78 & 5.47 & 5.63 & 5.95 & 17.06 & 6.47 & 8.42 & 6.08 & 4.56 & 25.53 & 7.39 & 8.56 & 12.38 & 28.33 \\
\hline$T_{3}$ & 4.61 & 6.49 & 5.80 & 16.91 & 6.39 & 6.59 & 5.78 & 18.77 & 7.20 & 8.50 & 6.52 & 4.62 & 26.84 & 7.42 & 9.16 & 12.54 & 29.12 \\
\hline $\mathrm{T}_{4}$ & 5.79 & 7.06 & 5.95 & 18.80 & 6.74 & 7.17 & 6.29 & 20.20 & 8.30 & 9.49 & 6.78 & 5.11 & 29.68 & 8.13 & 9.70 & 13.65 & 31.48 \\
\hline$T_{5}$ & 4.50 & 5.72 & 5.29 & 15.52 & 5.77 & 5.90 & 6.06 & 17.72 & 6.66 & 8.64 & 6.56 & 5.00 & 26.86 & 6.69 & 9.25 & 13.35 & 29.30 \\
\hline$T_{6}$ & 6.04 & 7.66 & 5.12 & 18.82 & 6.43 & 7.32 & 5.51 & 19.26 & 8.59 & 8.88 & 6.54 & 4.20 & 28.21 & 8.64 & 9.41 & 11.79 & 29.83 \\
\hline $\mathrm{T}_{7}$ & 5.87 & 7.44 & 5.37 & 18.68 & 6.63 & 6.93 & 5.30 & 18.85 & 9.02 & 9.05 & 6.68 & 4.45 & 29.20 & 9.31 & 9.47 & 11.83 & 30.62 \\
\hline $\mathrm{T}_{8}$ & 4.15 & 5.28 & 4.25 & 13.68 & 7.02 & 7.47 & 5.28 & 19.76 & 9.18 & 9.59 & 6.19 & 4.60 & 29.57 & 9.45 & 10.57 & 11.44 & 31.45 \\
\hline SEd & 0.20 & 0.29 & 0.22 & 0.73 & 0.29 & 0.31 & 0.24 & 0.83 & 0.38 & 0.40 & 0.30 & 0.24 & 1.30 & 0.36 & 0.41 & 0.50 & 1.30 \\
\hline $\mathrm{CD}(\mathrm{P}=0.05)$ & 0.47 & 0.62 & 0.47 & 1.60 & 0.61 & 0.66 & 0.52 & 1.80 & 0.81 & 0.85 & 0.60 & 0.51 & 2.70 & 0.78 & 0.87 & 1.07 & 2.72 \\
\hline
\end{tabular}

RDF- Recommended Dose of Fertilizer (150:50:50 N:P:K kg/ha-1) NS - Non-significant

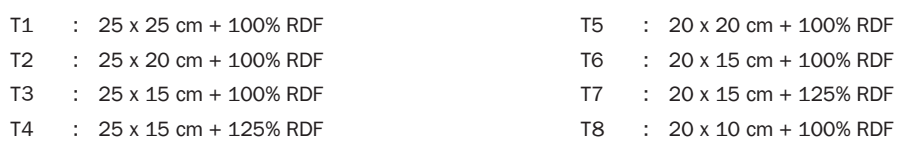

Higher nutrient uptake was recorded under the spacing level of $25 \mathrm{~cm} \times 15 \mathrm{~cm}$ fertilized with higher nutrient dose (125\% RDF) followed by the conventional system with 100\% RDF which might be due to higher dry-matter production and improved nutrient absorption brought out by increased nutrient dose application.

Table 4. Effect of high-density planting on potassium uptake $\left(\mathrm{kg} \mathrm{ha}^{-1}\right)$ of rice under the modified system of rice intensification

\begin{tabular}{|c|c|c|c|c|c|c|c|c|c|c|c|c|c|c|c|c|c|}
\hline \multirow{2}{*}{ Treatments } & \multicolumn{4}{|c|}{ Active tillering stage } & \multicolumn{4}{|c|}{ Panicle initiation stage } & \multicolumn{5}{|c|}{ Flowering stage } & \multicolumn{4}{|c|}{ Maturity stage } \\
\hline & Leaf & Culm & Root & Total & Leaf & Culm & Root & Total & Leaf & Culm & Root & Panicle & Total & Leaf & Culm & Panicle & Total \\
\hline $\mathrm{T}_{1}$ & 15.01 & 18.70 & 17.96 & 51.67 & 24.1 & 24.11 & 8.17 & 56.60 & 26.58 & 31.56 & 21.34 & 18.79 & 98.26 & 19.43 & 39.99 & 62.68 & 122.1 \\
\hline$T_{2}$ & 15.62 & 20.65 & 19.10 & 55.37 & 28.31 & 28.16 & 8.58 & 65.90 & 27.55 & 33.81 & 21.95 & 19.87 & 103.17 & 20.36 & 41.22 & 72.30 & 133.9 \\
\hline$T_{3}$ & 20.17 & 23.77 & 22.90 & 66.84 & 34.69 & 33.94 & 9.91 & 72.81 & 31.18 & 37.93 & 23.12 & 18.49 & 110.72 & 22.96 & 44.83 & 73.95 & 141.7 \\
\hline $\mathrm{T}_{4}$ & 24.63 & 27.16 & 23.79 & 75.58 & 37.72 & 37.65 & 11.76 & 76.08 & 33.99 & 44.29 & 25.14 & 21.76 & 125.18 & 25.67 & 51.75 & 78.63 & 156.0 \\
\hline$T_{5}$ & 18.37 & 23.66 & 22.21 & 64.23 & 33.92 & 29.08 & 11.09 & 69.25 & 28.17 & 38.89 & 23.56 & 20.36 & 110.98 & 18.95 & 45.92 & 77.31 & 142.2 \\
\hline $\mathrm{T}_{6}$ & 24.16 & 28.46 & 19.59 & 72.21 & 37.35 & 36.58 & 7.29 & 75.65 & 34.69 & 41.44 & 22.55 & 18.09 & 116.77 & 26.77 & 47.04 & 64.54 & 138.3 \\
\hline $\mathrm{T}_{7}$ & 23.82 & 27.32 & 21.17 & 72.31 & 35.03 & 35.65 & 8.03 & 73.57 & 36.08 & 42.18 & 23.22 & 18.44 & 119.92 & 30.75 & 48.27 & 68.76 & 147.8 \\
\hline $\mathrm{T}_{8}$ & 16.14 & 20.69 & 17.82 & 54.65 & 39.26 & 38.51 & 7.51 & 77.23 & 38.12 & 45.35 & 22.29 & 18.87 & 124.63 & 35.90 & 52.08 & 66.22 & 154.2 \\
\hline SEd & 0.92 & 1.10 & 0.86 & 2.90 & 1.10 & 1.20 & 0.92 & 3.20 & 1.55 & 1.84 & 0.92 & 0.83 & 5.20 & 1.10 & 1.90 & 2.70 & 5.7 \\
\hline$C D(P=0.05)$ & 1.97 & 2.30 & 1.85 & 6.10 & 2.40 & 2.50 & 1.97 & 6.90 & 3.32 & 3.90 & 1.97 & 1.77 & 11.20 & 2.40 & 4.10 & 5.80 & 12.3 \\
\hline
\end{tabular}

RDF- Recommended Dose of Fertilizer (150:50:50 N:P:K kg/ha-1)

NS - Non-significant

$\begin{array}{ll}\mathrm{T}_{1} & : 25 \times 25 \mathrm{~cm}+100 \% \mathrm{RDF} \\ \mathrm{T}_{2} & : 25 \times 20 \mathrm{~cm}+100 \% \mathrm{RDF} \\ \mathrm{T}_{3}: & 25 \times 15 \mathrm{~cm}+100 \% \mathrm{RDF} \\ \mathrm{T}_{4}: & 25 \times 15 \mathrm{~cm}+125 \% \mathrm{RDF}\end{array}$

More number of plants under conventional system compete for the available resources resulting in higher nutrient uptake. Lower nutrient uptake in the SRI method of planting can be attributed to the

$$
\begin{aligned}
& \mathrm{T}_{5}: 20 \times 20 \mathrm{~cm}+100 \% \text { RDF } \\
& \mathrm{T}_{6}: 20 \times 15 \mathrm{~cm}+100 \% \text { RDF } \\
& \mathrm{T}_{7}: 20 \times 15 \mathrm{~cm}+125 \% \mathrm{RDF} \\
& \mathrm{T}_{8}: 20 \times 10 \mathrm{~cm}+100 \% \text { RDF }
\end{aligned}
$$

lesser dry-matter production due to a lesser number of tillers $/ \mathrm{m}^{2}$. Higher nitrogen supply, enhanced drymatter accumulation by the improved synthesis of photo-assimilates in leaves and later partitioning 
of photo-assimilates to the reproductive organs (Ronanki et al., 2017). N uptake in leaf and culm showed a reduction from flowering to maturity stage resulting in lower radiation use efficiency and higher partitioning of assimilates to panicles (Bhavya et al., 2018). Higher N uptake by roots recorded under plant spacing of $25 \mathrm{~cm} \times 15 \mathrm{~cm}$ with $125 \% \mathrm{RDF}\left(\mathrm{T}_{4}\right)$ was due to higher $\mathrm{N}$ uptake at an increased dose of fertilization provided in the particular treatment. Phosphorus uptake by leaf, culm, and root was significantly higher at the plant spacing of $20 \mathrm{~cm}$ x $10 \mathrm{~cm}$ with 100\% RDF ( $\left.\mathrm{T}_{8}\right)$ preceded by $25 \mathrm{~cm} \mathrm{x}$ $15 \mathrm{~cm}$ spacing with $125 \% \operatorname{RDF}\left(T_{4}\right)$ due to greater production of dry-matter as a consequence of high uptake due to increased competition among the plants and application of higher dose of fertilizers. Higher uptake of potassium by leaf, culm and root at all growth stages under the plant spacing of $20 \mathrm{~cm} \times 10 \mathrm{~cm}$ with $100 \% \operatorname{RDF}\left(\mathrm{T}_{8}\right)$ and $25 \mathrm{~cm} \times$ $15 \mathrm{~cm}$ spacing with $125 \%$ RDF $\left(\mathrm{T}_{4}\right)$ also could be attributed to the improved potassium uptake from soil and higher dry-matter accumulation at higher fertilizer dose.

\section{CONCLUSION}

The experimental results revealed that higher dry-matter partitioning of leaf, culm, root and panicle were obtained under closer plant spacing of $20 \mathrm{~cm} \mathrm{x}$ $10 \mathrm{~cm}$ with 100\% RDF over other treatments while lower dry-matter accumulation was recorded at wider crop geometry of $25 \mathrm{~cm}$ x $25 \mathrm{~cm}$ with $100 \%$ RDF (SRI). The nutrient uptake recorded under $25 \times 15 \mathrm{~cm}$ with 125\% RDF was higher over other treatments followed by $20 \mathrm{~cm} \times 10 \mathrm{~cm}$ with $100 \%$ RDF while the SRI method of planting recorded lower nutrient uptake.

\section{REFERENCES}

Aggarwal, N. and A. Singh. 2015. Crop performance, nutrient uptake vis-a-vis weed suppressive ability of mechanically transplanted rice (Oryza sativa) as influenced by age of seedlings and planting density. Indian J. Agron., 60(2): 255-260.

Bhavya, M., D. Kumar and G. Girijesh. 2018. Root growth and tillering behavior of rice as influenced by systems of establishment. Int. J. Agric. Sci., 10(6): 5531-5535.

CPG. 2012. Crop Production Guide. Department of Agriculture, Govt. of Tamil Nadu, Chennai and Tamil Nadu Agricultural University, Coimbatore.

Dass, A. and S. Chandra. 2013. Irrigation, spacing and cultivar effects on net photosynthetic rate, dry-matter partitioning and productivity of rice under system of rice intensification in Mollisols of northern India. Experimental Agric., 49(4): 504-523.

Gomez, K.A. and A.A. Gomez, 2010.Statistical Procedures for Agricultural Research. $2^{\text {nd }}$ Ed. Wiley India Pvt Ltd., New Delhi, India.

Najeebullah and Chinnusamy, C. 2017. Influence of plant density, fertilizer levels and foliar nutrition on growth and yield of irrigated blackgram. Madras Agric. J., 104 (10-12): 345-349.

NRRI. 2018. ICAR-National Rice Research Institute's Annual Report 2017-18. Cuttack, Odisha, India.

Ronanki, S., A. Madhavi, R. Reddy and G. Sreenivas. 2017. Dry-matter accumulation, partitioning and nitrogen uptake of transplanted rice under varied plant densities and nitrogen levels. Chemical Sci. Review Letters, 6(23): 1975-1979.

Thakur, A.K., S. Rath, S. Roychowdhury and N. Uphoff. 2010. Comparative performance of rice with System of Rice Intensification (SRI) and conventional management using different spacings. J. Agron. Crop Sci., 196: 146-159.

Thavaprakaash, N., P. Baskar and A. Velayutham. 2017. Influence of planting geometry on growth and yield of rice (Oryza sativa L.) under SRI practices. The Bioscan, 12(1): 557-560. 